\title{
Is open surgery for iatrogenic esophageal perforation now a surgical relic, like bloodletting and trepanation?
}

\author{
Katie S. Nason, MD, MPH
}

See related article on pages $1550-5$.

Open surgery for iatrogenic esophageal perforation has long been the standard of care for patients with evidence of extraluminal contamination, including contrast extravasation, pleural effusions, or sepsis. During the past decade, however, endoluminal stenting has become increasingly popular, despite a lack of prospective studies and heterogeneity among treatment groups. In their article in this issue of the Journal, "A Propensity-Matched Comparison of Cost and Outcomes Following Esophageal Stent Placement or Primary Surgical Repair for an Iatrogenic Esophageal Perforation," Freeman and colleagues $^{1}$ seek to provide further evidence supporting the safety, efficacy, and cost of an endoluminal approach to acute intrathoracic iatrogenic esophageal perforation. In a propensity-matched comparison with surgical patients, operative mortality was similar and morbidity, mean stay, time to oral intake, and cost were significantly lower in the esophageal stent cohort. Freeman and colleagues ${ }^{1}$ conclude that esophageal stenting for acute esophageal perforation appears to be as effective as surgical repair.

The article addresses an important question for thoracic surgeons, and the findings are promising. The data demonstrate that patients with iatrogenic esophageal perforation can be safely and effectively managed with a comprehensive approach that includes endoluminal stenting for immediate leak diversion and minimally invasive drainage of intrathoracic and mediastinal contamination. Similar to published results regarding the use of esophageal stents for management of intrathoracic esophagogastric anastomotic leak, ${ }^{2}$ leak control is high,

From the Department of Cardiothoracic Surgery, University of Pittsburgh, Pittsburgh Pa.

K.S.N.'s work is supported by Award No. K07CA151613 from the National Cancer Institute. The content is solely the responsibility of the author and does not necessarily represent the official views of the National Cancer Institute or the National Institutes of Health.

Disclosures: Author has nothing to disclose with regard to commercial support.

Received for publication Feb 12, 2015; accepted for publication Feb 12, 2015; available ahead of print March 24, 2015.

Address for reprints: Katie S. Nason, MD, MPH, University of Pittsburgh, 5200 Centre Ave, Suite 715, Shadyside Medical Building, Pittsburgh, PA 15232

(E-mail: nasonks@upmc.edu).

J Thorac Cardiovasc Surg 2015;149:1556-7

$0022-5223 / \$ 36.00$

Copyright (c) 2015 by The American Association for Thoracic Surgery

http://dx.doi.org/10.1016/j.jtcvs.2015.02.028 and time to eating and major morbidity are reduced in patients with stents. In this study, Freeman and colleagues ${ }^{1}$ focus specifically on management of iatrogenic intrathoracic perforation, which strengthens the study by eliminating the heterogeneity introduced with multiple etiologies. Another study strength is the use of propensity matching in an attempt to reduce confounding. Propensity matching is used to create 2 groups that are well matched with respect to factors that might affect treatment decisions. In this study, age, sex, comorbid diseases, time to intervention, and sepsis at treatment are included as propensity-matching variables. There are, however, additional factors that influence the decision for operative intervention, including the size of the injury, location of the injury (distance from the incisors), presence of underlying esophageal disorders (such as motility disorders, strictures, and achalasia), and the degree of mediastinal and intrathoracic contamination All these are unaccounted for in the matching; as such, they represent unmeasured factors influencing surgical decisions regarding management. It is premature to interpret definitively the higher mortality, morbidity, cost, and stay with surgery as a direct result of treatment choice so long as unmeasured differences in the patient's perforation-associated condition are not accounted for in propensity-matching or outcomes analysis. With larger numbers of patients and a more inclusive data set, incorporation of these additional variables into propensity matching and adjustment of the relationship between treatment and outcomes for these confounding variables in multivariable analysis would help to identify the independent prognostic factors associated with cost, morbidity, and mortality. In light of the small numbers and retrospective design, these analyses were not possible in the study of Freeman and colleagues. ${ }^{1}$ Finally, another limitation of the current study is the binary designation for morbidity (yes/no); this clusters all patients into 2 groups without consideration of the numbers or types of morbidity encountered. As such, a superficial wound infection and septic shock would be considered equivalent with regard to the morbidity outcome but would clearly have different implications for the patient.

Despite these words of caution, the data are compelling and warrant examination of the findings of other investigators. Dasari and colleagues ${ }^{3}$ examined the role of esophageal stents with a systematic literature review and 
found that stenting is technically feasible, safe, and effective when performed along with mediastinal or pleural drainage. A meta-analysis of the management of esophageal perforations by Biancari and associates ${ }^{4}$ also showed that stentgrafting was associated with lower mortality, but they cautioned that studies may be biased by patient selection and limited experience. Iatrogenic perforation was the etiology in $13.2 \%$. A higher percentage of patients with iatrogenic perforation were analyzed $(67 \%)$ in a study by BenDavid and associates ${ }^{5}$; all were safely treated within 24 hours of injury with a covered removable stent. Cost analysis with use of stents revealed a cost similar to that with stenting in the study of Freeman and colleagues. ${ }^{1}$ Finally, in another recent multicenter study by Biancari and colleagues ${ }^{6}(36 \%$ iatrogenic perforations), propensity-adjusted analysis, which included perforation-specific details including location and size, showed that morbidity and mortality were not statistically different in the stent group relative to the surgically treated group.

Taken together with these previous studies, the data for management of iatrogenic perforation increasingly favor early intervention with esophageal stenting. As noted by Dasari and colleagues ${ }^{3}$ and Ben-David and associates, ${ }^{5}$ aggressive thoracoscopic drainage of intrathoracic and mediastinal contamination and early enteral nutritional support are critical to the process. Optimally, stents are actively managed, with frequent imaging, repeated endoscopy when needed, and a low threshold for surgical intervention if leak containment cannot be achieved. Through prospective institutional algorithms, such as that proposed by Ben-David and associates, ${ }^{5}$ the combination of endoluminal and minimally invasive intracavitary approaches will likely lead to reduced cost, reduced associated morbidity and mortality, and improved long-term outcomes.

\section{References}

1. Freeman RK, Herrera A, Asciti AJ, Dake M, Mahidhara RS. A propensity matched comparison of cost and outcomes after esophageal stent placement or primary surgical repair for iatrogenic esophageal perforation. J Thorac Cardiovasc Surg. 2015;149:1550-5.

2. Schaheen L, Blackmon SH, Nason KS. Optimal approach to the management of intrathoracic esophageal leak following esophagectomy: a systematic review Am J Surg. 2014;208:536-43.

3. Dasari BV, Neely D, Kennedy A, Spence G, Rice P, Mackle E, et al. The role of esophageal stents in the management of esophageal anastomotic leaks and benign esophageal perforations. Ann Surg. 2014;259:852-60.

4. Biancari F, D'Andrea V, Paone R, Di Marco C, Savino G, Koivukangas V, et al. Current treatment and outcome of esophageal perforations in adults: systematic review and meta-analysis of 75 studies. World J Surg. 2013:37:1051-9.

5. Ben-David K, Behrns K, Hochwald S, Rossidis G, Caban A, Crippen C, et al Esophageal perforation management using a multidisciplinary minimally invasive treatment algorithm. J Am Coll Surg. 2014;218:768-74.

6. Biancari F, Saarnio J, Mennander A, Hypén L, Salminen P, Kuttila K, et al Outcome of patients with esophageal perforations: a multicenter study. World J Surg. 2014;38:902-9. 